\title{
Qualidade da água de um ambiente lótico sob impacto antropogênico e sua comunidade bentônica
}

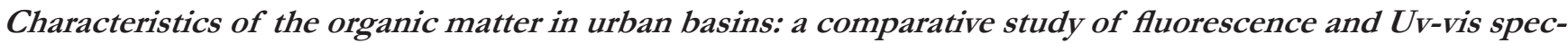 troscopy in river and lake}

\section{Carla Cristina Bem ${ }^{1}$; Janet Higuti² e Júlio César Rodrigues de Azevedo ${ }^{3}$}

\author{
${ }^{1}$ Aluna de doutorado do Programa de Pós-graduação em Engenharia de Recursos Hídricos e Ambiental pela Universidade \\ Federal do Paraná (UFPR), Curitiba - PR, Brasil \\ carla.dhs@ufpr.br
}
${ }_{2}^{2}$ Pesquisadora do Núcleo de Pesquisas em Limnologia, Ictiologia e Aqüicultura e do Programa de Pós-graduação em Ecologia de Ambientes Aquáticos pela Universidade Estadual de Maringá (UEM), Maringá - PR, Brasil
higuti@nupelia.uem.br
${ }^{3}$ Professor da Universidade Tecnológica Federal do Paraná (UTFPR) e Professor Associado do Programa de Pós-graduação em Engenharia de Recursos Hídricos e Ambiental pela Universidade Federal do Paraná (UFPR), Curitiba - PR, Brasil

jcrazevedo@utfpr.edu.br

Recebido: 26/01/14 - Revisado: 05/09/14 - Aceito: 05/12/14

\section{RESUMO}

Este estudo teve como objetivo avaliar a qualidade da água e o uso de macroinvertebrados bentônicos como ferramenta auxiliar para a gestão de recursos bidricos tendo como área de estudo a Bacia do Rio Iguaçu. Ao longo do rio Iguaç foram amostrados dezesseis pontos, contemplando sua nascente até a foz. A amostragem do sedimento foi realizada com uma draga de Petersen modificada, os organismos foram triados e fixados com álcool $70 \%$. Os resultados evidenciaram uma baixa riquez̧a taxonômica (nove táxons), sendo os grupos Chironomidae e Oligochaeta os mais frequentes durante o monitoramento. Devido a baixa riqueza de táxons a partir de grandes grupos não foi possivel fazer inferências em relação a qualidade da água. Por outro lado, a identificação dos gêneros de Chironomidae, corroborou com os resultados dos parâmetros abióticos da qualidade da água. Nos pontos poluidos foram observados somente os gêneros Chironomus e Polypedilum, contudo, uma melhora da qualidade da água foi observada ao longo do rio Iguaçu através da alteração dos gêneros de Chironomidae (por exemplo, Orthocladiinae) e o aumento de riqueza. A avaliação dos macroinvertebrados bentônicos permitiu verificar a variação temporal da qualidade da água, resultado que possui grande significado para a gestão de recursos hidricos, devido a periodicidade das amostragens e consequente falta de dados que expliquem variaçoes não identificadas por amostragens pontuais.

Palavras Chave: Biomonitoramento. Macroinvertebrados. Rio

\begin{abstract}
The main goal of this study is to evaluate water quality and the application of benthic macroinvertebrates as tools to assist the management of water resources in the Iguacu River Basin. Sixteen points were sampled along the Iguacu River (source to mouth). The sediment sampling was performed with a modified Petersen grab, the organisms were sorted and fixed with 70\% alcohol. The results showed low species richness (nine taxa), and Chironomidae and Oligochaeta were the groups most frequently found during monitoring. Due to the lower number of taxa from large groups it was not possible to infer the water quality. Moreover, identifying the level of genera of Chironomidae, corroborated the results of the abiotic parameters of water quality. At the polluted points only genera Polypedilum and Chironomus were found, however an improvement in water quality was observed along the Iguacu River through changes in Chironomidae genera (eg Orthocladiinae) and the increase of richness. The assessment of benthic macroinvertebrates demonstrated the temporal variation of water quality, a result which has great significance for the management of water resources, because of the frequency of sampling and consequent lack of data explaining variations not identified by point sampling.
\end{abstract}

Keywords: Biomonitoring. Macroinvertebrates. Rivers 


\section{INTRODUÇÃO}

O crescimento acelerado de unidades legislativas em muitos casos não ocorre com planejamento, de modo que esta expansão urbana desordenada contribui para a degradação do meio ambiente. Em relação as alterações da qualidade da água, os principais impactos são originados a partir da poluição por fontes pontuais e difusas que lançam vários poluentes que interagem de maneira específica no meio ambiente.

Antes a gestão de recursos hídricos limitava-se somente a matéria orgânica e nutrientes e, atualmente, a hormônios, fármacos em geral, hidrocarbonetos e metais pesados no meio ambiente. Substâncias estas que em sua maioria são recalcitrantes, com características de biomagnificação e bioacumulação, causando diversos impactos no ecossistema e na cadeia trófica.

A compreensão da complexidade das interações ambientais é possível apenas por meio de um olhar holístico, o qual considera as interações entre alterações físicas, químicas e a biota, sendo necessário contemplar além da fotografia, consagrada no monitoramento ambiental. Usualmente as redes de monitoramento da qualidade da água analisam parâmetros físico, químicos e hidráulicos como a vazão normalmente, não avaliando a integridade do ecossistema, isto é, a qualidade da água como resultado das interações ecológicas que ocorrem entre a biota e desta com o ambiente abiótico geram o estado ecológico da qualidade da água.

A legislação ambiental brasileira não considera o estado ecológico da água, ao contrário de alguns países como os Estados Unidos que em 1972 instituíram "Clean Water Act” e em países pertencentes a União Européia, que estão implementando "The Water Framework Directive" proposto em 2000 com horizonte de execução para 2015.

A legislação brasileira faz menções ao meio ambiente na Constituição Federal de 1988 que deliberou no Capítulo 6, Art. 225 que "todos têm direito ao meio ambiente ecologicamente equilibrado" (BRASIL, 2012a). Entretanto apenas a resolução do Conselho Nacional do Meio Ambiente (CONAMA), $N^{\circ}$ 357/2005 (BRASIL, 2012b) define os parâmetros de qualidade dos corpos aquáticos, as diretrizes ambientais para seu enquadramento e inserem referências as questões ecológicas sem, no entanto, considerá-la como aspecto normativo.

A preocupação com a biota aquática é apenas prescrita para substâncias potencialmente tóxicas e interações entre estas de forma que "não poderão conferir às águas características capazes de causar efeitos letais ou alteração de comportamento, reprodução ou fisiologia da vida".

Impondo que os critérios de avaliação para as classes e usos previstos devam ser realizados apenas por parâmetros físicos, químicos e bacteriológicos, não considerando indicadores biológicos, além de outros processos que ocorrem continuamente no meio ambiente como fluxo e balanço de energia, produtividade, estrutura e funções do ecossistema (LUZ; FERREIRA, 2011), os quais estão envolvidos nos ciclos de nutrientes, consumo e produção de matéria orgânica, degradação, assimilação de contaminantes e na consideração do corpo aquático como ser saudável.
A avaliação da integralidade do meio ambiente, em especial dos recursos hídricos considera além da regulação de concentrações, a presença e ausência de substâncias e propõe que o ambiente também seja analisado em termos dos organismos que estão ou deveriam estar presentes. Teoricamente qualquer organismo pode ser utilizado como bioindicador incluindo diversos protocolos para avaliação de bactérias, fungos, protozoários (conhecido como sistema sapróbico), algas, macroinvertrados, macrófitas e peixes. A escolha do bioindicador será em função do objetivo do monitoramento ou do contaminante que se pretende avaliar.

Um dos aspectos positivos do monitoramento biológico para a avaliação ambiental é que a resposta biológica fornece informações temporais sobre a qualidade da água, além da interação dos organismos com parâmetros físico-químicos. Outro aspecto importante que se deve considerar na análise biológica, é o fato de sua determinação ser mais econômica em comparação ao monitoramento tradicional. O monitoramento biológico pode ser realizado com foco em várias aplicações, para isso, entretanto é necessário amplo conhecimento da área de estudo, amostragens em diferentes rios para que haja representatividade de um grande espectro quantitativo e qualitativo da água em relação aos organismos presentes.

Dentre os vários indicadores biológicos existentes, os macroinvertebrados bentônicos são os mais utilizados devido ao fato de possuírem alta diversidade taxonômica, sendo representados, principalmente por Arthtopoda (Insecta, Acarina e Crustacea), Mollusca (Gastropoda e Bivalvia) e Annelida (Oligochaeta e Hirudinea). Algumas espécies de macroinvertebrados bentônicos possuem maior sensibilidade a perturbações enquanto outros são mais tolerantes. Além de a fauna bentônica possuir papel importante na dinâmica de nutrientes e na transformação da matéria orgânica tanto de ambientes lênticos como lóticos, promover o biorrevolvimento do sedimento superficial liberando assim nutrientes para a coluna d'água e aeração do sedimento (REZENDE; GONÇALVES; PETRUCIO, 2010).

Os principais aspetos que influenciam a distribuição de macroinvertebrados bentônicos são as características físicas e químicas da água (concentração de matéria orgânica, nutrientes), tipo de substrato e características hidrológicas (ARTHINGTON et al., 2010; BRACCIA; VOSHELL-JR, 2006; BUSS et al., 2004; BUSS et al., 2002; DORNFELD; ESPINDOLA; LEITE, 2005; NIYOGI et al., 2007; RHEA et al., 2006; SILVEIRA et al., 2006; ).

A escolha de macroinvertebrados bentônicos em monitoramentos biológicos deve-se por serem: amplamente distribuídos, abundantes e de fácil coleta; possuem mobilidade restrita ou são sedentários; caracterizam a água em escalas temporais; permitem a avaliação de processos de bioacumulação e biomagnificação por participarem da cadeia de detritos (HARE, 1992; HARE; CAMPBELL, 1992, LYNCH; POPP; JACOBI, 1988; MYSLINSKI; GINSBURG, 1977; ROSENBERG; WIENS, 1976; YSEBAERT et al., 2003).

No biomonitoramento de macroinvertebrados bentônicos alterações na estrutura das comunidades bentônicas ocasionadas por poluição provoca redução de diversidade de espécies em relação a áreas de referência e aumento da abundância de 
espécies tolerantes a poluição (HEPP; SANTOS, 2009). Além da presença de metais (DOMINGUES et al., 2007), e matéria orgânica (ARTHINGTON et al., 2010), outros compostos também podem afetar a fauna aquática como os contaminantes emergentes: antiinflamatórios não esteriodais, drogas hipolepemiantes, antibióticos, hormônios sexuais femininos (HSFs), antiepiléticos, bloqueadores beta, antidepressivos, antineoplásicos e meios de contraste para raio-x (SANTOS et al., 2010).

Para a gestão de recursos hídricos é interessante que o biomonitoramento seja aplicado de modo a avaliar a comunidade presente, para que os organismos coletados possam inferir o comprometimento da qualidade da água e dessa forma a partir de observações avaliar relações com interferentes, sendo que os macroinvertebrados bentônicos devido a sua mobilidade restrita ou sedentária permitem inferir a qualidade da água em estações de monitoramento.

De acordo com Josefsson e Baaner (2011), a possibilidade de avaliar os ecossistemas de acordo com parâmetros biológicos como "elementos de qualidade", e sua presença ou ausência, pode ser importante para os gestores, mas por informações de condensação em um dos parâmetros, a capacidade para diagnosticar sintomas ecológicos é perdida. Dessa forma, nenhuma informação real de diagnóstico é fornecida pelo fato de que algumas espécies serem raras ou ausentes em um local potencialmente afetado, em comparação com um local de referência, pois as respostas biológicas reagem de forma única em cada ambiente, sendo necessário avaliar os efeitos individuais ou sinérgicos que ocorrem na área de interesse.

Deve ser considerado que o biomonitoramento neste contexto busca além de fornecer dados, auxiliar na construção de ferramentas para a melhoria da qualidade ambiental, sendo que a qualidade ambiental deve considerar que no caso das comunidades biológicas, o objetivo não deve ser de retornar as condições de referência, mas sim, reabilitar a capacidade de um ambiente perturbado aproximar-se da trajetória anterior (HARRIS et al., 2006), isto é próximo de seu estado original.

No Brasil foram realizados alguns estudos importantes sobre a aplicação de macroinvertebrados bentônicos. Moreno et al. (2009) desenvolveram uma metodologia para o uso de indicadores biológicos, baseando-se na hipótese que o gradiente de poluição altera a estrutura e a composição da comunidade de macroinvertebrados bentônicos por meio de um índice multimétrico. Porém para elaboração e aplicação de índices como este é necessário que existam redes de monitoramento contínuo de qualidade e quantidade da água, as quais são poucas, praticamente não há monitoramento do sedimento e tão pouco biológico, exceto o realizado pela Companhia Ambiental do Estado de São Paulo (CETESB).

Outro estudo importante realizado no Brasil que apresentou caráter vanguardista é o Projeto Manuelzão, realizado pela Universidade Federal de Minas Gerais (UFMG), o qual desde 1997 busca melhorias nas condições ambientais na Bacia do Rio das Velhas e atua em diversas frentes como geoprocessamento e recuperação de matas ciliares e biomonitoramento, possuindo como um dos principais objetivos consolidar a "volta do peixe" e atingir níveis de qualidade de águas Classe 2 de acordo com o CONAMA 357/05 no trecho presente em região metropolitana
(FERREITA et al., 2012).

Uma das dificuldades encontradas na recuperação de rios é fato de muitos situarem-se em áreas densamente povoadas e as alterações de qualidade da água são localizadas próximos de suas nascentes, o que faz com que o rio perca sua fauna original e o gradiente para avaliação é invertido, isto é, de degradado a limpo.

Considerando estes aspectos, este trabalho teve como objetivo avaliar a aplicabilidade do uso de macroinvertebrados bentônicos como ferramenta complementar na gestão dos recursos hídricos com foco na avaliação da qualidade da água, tendo como área de estudo a Bacia do rio Iguaçu, a qual apresenta características de ambientes com diferentes níveis de comprometimento da qualidade da água e impactos a partir de sua nascente.

\section{MATERIAL E MÉTODOS}

\section{Área de Estudo}

O rio Iguaçu é o maior rio do estado do Paraná, a Bacia do rio Iguaçu devido ao seu tamanho foi dividida em 3 sub-bacias (Alto Iguaçu, Médio Iguaçu e Baixo Iguaçu). O rio Iguaçu corre em sentido leste a oeste, tendo sua nascente localizada próximo ao município de Curitiba e sua foz na cidade de Foz do Iguaçu, é formado pelo encontro dos rios Iraí e Atuba. Percorre cerca de $1.100 \mathrm{~km}$ com alguns trechos demarcando a divisa entre os estados de Santa Catarina e Paraná. Ao longo de seu percurso possui um desnível de $800 \mathrm{~m}$ e com uma considerável área de drenagem em torno de $70.800 \mathrm{~km}^{2}$ no território nacional e cerca de $1.837 \mathrm{~km}^{2}$ na Província de Missiones, Argentina. Cinco reservatórios de produção hidrelétrica estão localizados neste rio.

No rio Iguaçu foram monitorados dezesseis estações de amostragem. A localização geográfica, e o mapa com a localização espacial dos pontos amostrados estão apresentados na tabela 1 e figura 1 . Neste estudo, o rio foi amostrado desde sua nascente até próximo de sua foz no rio Paraná. Conforme apresentado na figura 1, a Bacia do Alto Iguaçu encontra-se inserida na Região Metropolitana de Curitiba (RMC), região com pequeno desnível do rio e grande sinuosidade, com uma área de drenagem de aproximadamente $3.000 \mathrm{~km}^{2}$ (até a seção fluviométrica da Estação Balsa Nova, localizado no município de Balsa Nova).

A população total da bacia do Alto Iguaçu é de aproximadamente três milhões de habitantes, da qual $92 \%$ caracteriza-se como população urbana. A RMC está passando por um processo de ocupação irregular de várzeas e áreas de mananciais, em especial na margem direita do Rio Iguaçu, ocasionando problemas acerca dos sistemas de abastecimento de água, do tratamento de esgotos sanitários e dos sistemas de drenagem urbana, os quais não acompanham o crescimento das cidades.

Tanto a bacia do Iguaçu Médio como o Baixo apresentam menor impacto de fontes pontuais, embora nestas duas sub-bacias o impacto difuso seja predominante, devido as características agrícolas da economia dos municípios localizados nesta área. 


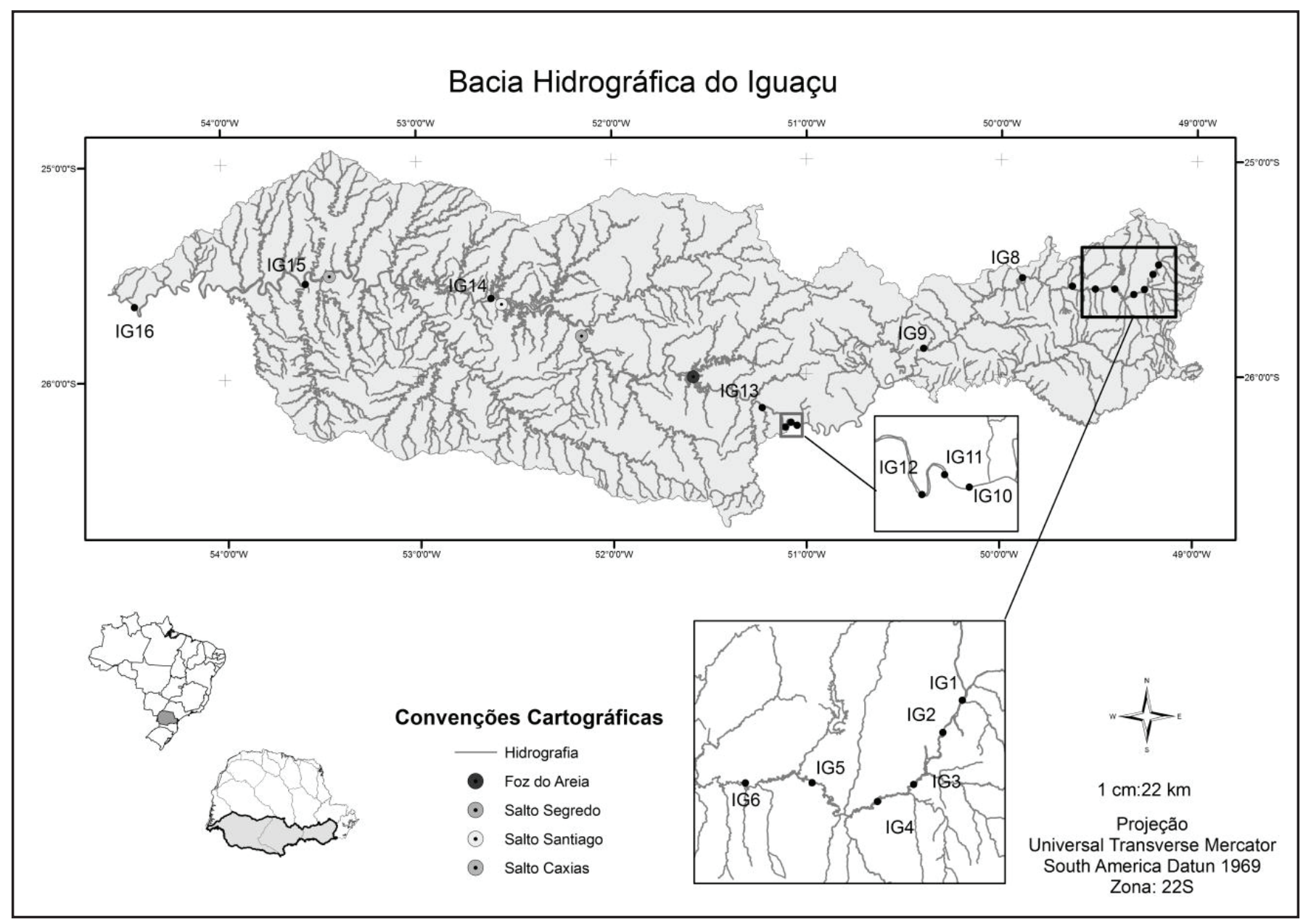

Figura 1 - Bacia do Alto Iguaçu, com localização dos pontos de monitoramento

Tabela 1 - Localização geográfica dos pontos amostrados no rio Iguaçu

\begin{tabular}{|c|c|c|c|}
\hline PONTO & \multicolumn{2}{|c|}{ COORDENADA (UTM SAD 69) } & LOCAL \\
\hline$\overline{I G-1}$ & $\mathrm{O} 49^{\circ} 11^{\prime} 25.1^{\prime \prime}$ & S 25 29' 3.3”' & BR 277 \\
\hline IG-2 & O 49० 13' 7.9'” & S $25^{\circ} 31^{\prime} 41.7^{\prime \prime}$ & Parque Naútico \\
\hline IG-3 & $\mathrm{O} 49^{\circ} 15^{\prime} 41.6^{\prime \prime}$ & $\mathrm{S} 25^{\circ} 35^{\prime} 57.1^{\prime \prime}$ & Ponte Umbarazinho \\
\hline IG-4 & O 49० $18^{\prime} 55.9^{\prime \prime}$ & $\mathrm{S} 25^{\circ} 37^{\prime} 22.1^{\prime \prime}$ & BR 116, Fazenda Rio Grande \\
\hline IG-5 & $\mathrm{O} 49^{\circ} 24^{\prime} 48.8^{\prime \prime}$ & $\mathrm{S} 25^{\circ} 35^{\prime} 54.3^{\prime \prime}$ & Parque das Pontes, Araucária \\
\hline IG-6 & $\mathrm{O} 49^{\circ} 30^{\prime} 47.8^{\prime \prime}$ & $\mathrm{S} 25^{\circ} 35^{\prime} 59.7^{\prime \prime}$ & Guajuvira \\
\hline IG-7 & O 49 37' 52.7’" & $\mathrm{S} 25^{\circ} 35^{\prime} 15.1^{\prime \prime}$ & Balsa Nova \\
\hline IG-8 & O 49 $53^{\prime} 20.0^{\prime \prime}$ & S $25^{\circ} 32^{\prime} 59.4^{\prime \prime}$ & Porto Amazonas \\
\hline IG-9 & O $50^{\circ} 23^{\prime} 42.3^{\prime \prime}$ & S 25 52' 52.7”' & São Mateus do Sul \\
\hline IG-10 & O $51^{\circ} 4^{\prime} 47.8^{\prime \prime}$ & $\mathrm{S} 26^{\circ} 9^{\prime} 32.0^{\prime \prime}$ & União da Vitória (ETA) \\
\hline IG-11 & O $51^{\circ} 2^{\prime} 53.9^{\prime \prime}$ & S $26^{\circ} 14^{\prime} 27.6^{\prime \prime}$ & União da Vitória (Centro) \\
\hline IG-12 & O $51^{\circ} 6^{\prime} 31.8^{\prime \prime}$ & S $26^{\circ} 14^{\prime} 58.2^{\prime \prime}$ & União da Vitória (ETE) \\
\hline IG-13 & 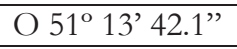 & $\mathrm{S} 26^{\circ} 9^{\prime} 32.0^{\prime \prime}$ & Porto Vitória \\
\hline IG-14 & O 52 37' 20.4”' & $\mathrm{S} 25^{\circ} 38^{\prime} 29.5^{\prime \prime}$ & \\
\hline IG-15 & O 53⒊ 34'32.9”' & S $25^{\circ} 33^{\prime} 47.9^{\prime \prime}$ & Ponte BR 163 \\
\hline IG-16 & O 54 27' 21.7”' & $\mathrm{S} 25^{\circ} 39^{\prime} 10.1^{\prime \prime}$ & PNI - Macuco Safari \\
\hline
\end{tabular}

Legenda: ETE (estação de tratamento de esgoto), ETA (estação de tratamento de água), PNI (Parque Nacional do Iguaçu) 


\section{METODOLOGIA}

Foram realizadas 4 coletas de amostras de água e sedimento (julho e novembro de 2012 e fevereiro e setembro de 2013). O monitoramento da fauna bentônica foi realizado pela coleta de amostras em triplicata com o auxilio de uma draga de Petersen modificada $\left(0,0180 \mathrm{~m}^{2}\right)$. As amostras de sedimento foram pré-triadas pela lavagem do sedimento em um jogo de peneiras (com abertura de malha de 2,0, 1,0 e 0,2 mm). O material retido na abertura de $0,2 \mathrm{~mm}$ foi acondicionado em frascos e preservados com álcool $70 \%$ para posterior triagem dos organismos sob microscópio estereoscópico, e identificação ao nível de gênero para a família Chironomidae de acordo com a chave de identificação elaborada por Trivinho-Strixino (2011). A identificação ao nível de gênero foi adotada por ser possível inferir características em relação à qualidade da água, sendo que a identificação ao nível de morfo-espécie é dificultosa devido a muitos organismos não estarem descritos e de acordo com Milosevic et al. (2014), a identificação do gênero não produz perda de informação em relação a morfo-espécie.

Nas amostras da água foram medidos parâmetros in situ (oxigênio dissolvido, $\mathrm{pH}$, condutividade, sólidos totais dissolvidos) com a sonda multiparamétrica marca Hanna (modelo HI 9828). Os demais parâmetros foram analisados de acordo com as metodologias propostas pelo Standard Methods for the examination of Water and Wastewater (APHA, 1998), exceto o carbono orgânico total que foi determinado pelo método de combustão da matéria orgânica no equipamento HiperTOC, de acordo com metodologia proposta pelo fabricante (THERMO ELECTRON CORPORATION, 2005). Os dados de vazão foram obtidos por meio de estações monitoradas pela Copel, Instituto das Águas do Paraná e Agência Nacional de Águas.

A avaliação da influência dos parâmetros analisados sobre os gêneros de Chironomidae foi realizada pela técnica de regressão linear múltipla (RLM), com o objetivo de identificar qual o parâmetro (físico, químico ou hidráulico) tem maior correlação com os gêneros identificados, isto é, maior influência sobre o estabelecimento do organismo no ponto amostrado.

\section{RESULTADOS E DISCUSSÃO}

\section{Fauna bentônica}

Durante o período do monitoramento foram coletados 1132 organismos distribuídos em nove grupos taxonômicos. Chironomidae e Oligochaeta foram os grupos mais representativos em abundância (Tabela 2). Entre os pontos IG-1 a IG-8 foram registrados maior riqueza de táxons, no entanto, foram encontrados táxons resistentes e relacionados com contaminação de esgoto como Nematoda (SILVA, 2009), além de Hydrachnidae.

A baixa riqueza de táxons de macroinvertebrados bentônicos no rio Iguaçu pode ser devido ao gradiente de poluição do rio, que possui comprometimento de sua qualidade desde sua nascente colaborando para que organismos resistentes se estabeleçam com sucesso, mesmo próximo de sua foz não houve ocorrência de táxons sensíveis a poluição da água, este fato provavelmente está relacionada à hidrodinâmica do rio, por exemplo, vazão, velocidade de corrente, além de maior profundidade e largura.

Os resultados do presente estudo evidenciaram que os macroinvertebrados identificados em escala taxonômica ao nível de grandes grupos não foram suficientes para serem utilizados como indicadores de qualidade da água, ao contrário dos estudos realizados por Buss et al. (2002), Hepp e Santos (2009) e Mophin -Kani e Murugesan (2014), os quais utilizaram grandes grupos para inferir características de qualidade da água. Neste sentido, faz-se necessário a identificação em nível taxonômico inferior, como gênero ou espécie quando possível, principalmente, os táxons com ampla distribuição entre os pontos de amostragem e que possuem organismos com diferentes níveis de tolerância a alterações na qualidade da água.

Tabela 2 - Número absoluto de macroinvertebrados bentônicos identificados no Rio Iguaçu

\begin{tabular}{|c|c|c|c|c|c|c|c|c|c|}
\hline \multirow{2}{*}{ Pontos } & \multicolumn{9}{|c|}{ Táxons } \\
\hline & Chironomidae & Oligochaeta & Nematode & Hydrachnidae & Bivalve & Haparticoida & Cyclopoida & Coleoptera & Ceratopogonidae \\
\hline IG-1 & 2 & 64 & 17 & 19 & & & & & \\
\hline IG-2 & 10 & 21 & 16 & 6 & & & & & \\
\hline IG-3 & 3 & 1 & 10 & 2 & & & & & \\
\hline IG-4 & 1 & 82 & & 3 & & & & & \\
\hline IG-5 & 1 & 112 & 2 & 1 & & & & & \\
\hline IG-6 & 6 & 36 & & 6 & & & & & \\
\hline IG-7 & 2 & 32 & & & & 6 & 5 & & \\
\hline IG-8 & 136 & 0 & 27 & & & & & & \\
\hline IG-9 & 16 & 22 & & & & & & & \\
\hline IG-10 & & 45 & & & & & & & \\
\hline IG-11 & 2 & 12 & & & & & & & \\
\hline IG-12 & 2 & 20 & & & & & & & \\
\hline IG-13 & 4 & & & & & & & & \\
\hline IG-14 & & 1 & & & 10 & & & & \\
\hline IG-15 & 81 & 16 & & & 9 & & & & \\
\hline IG-16 & 277 & 5 & & & 4 & & & 1 & 7 \\
\hline Total & 543 & 469 & 41 & 37 & 23 & 6 & 5 & 1 & 7 \\
\hline
\end{tabular}


Tabela 3 - Densidade (ind $/ \mathrm{m}^{2}$ ) de gêneros de Chironomidae ao longo do rio Iguaçu

\begin{tabular}{|c|c|c|c|c|}
\hline Ponto & Família & Gênero & Número & Densidade \\
\hline IG-1 & Chironominae & Chironomus & 2 & 37,0 \\
\hline IG-2 & Chironominae & Chironomus & 10 & 185,2 \\
\hline \multirow{2}{*}{ IG-3 } & \multirow{2}{*}{ Chironominae } & Chironomus & 2 & 37,0 \\
\hline & & Polypedilum & 1 & 18,5 \\
\hline IG-4 & Chironominae & Chironomus & 1 & 18,5 \\
\hline IG-5 & Chironominae & Polypedilum & 1 & 18,5 \\
\hline IG-6 & Chironominae & Polypedilum & 6 & 111,1 \\
\hline IG-7 & Chironominae & Polypedilum & 2 & 37,0 \\
\hline \multirow{3}{*}{ IG-8 } & \multirow{2}{*}{ Chironominae } & Polypedilum & 128 & 2370,4 \\
\hline & & Chironomus & 1 & 18,5 \\
\hline & Orthocladiinae & Thienemanniella & 7 & 129,6 \\
\hline \multirow{5}{*}{ IG-9 } & \multirow{3}{*}{ Chironominae } & Axarus & 1 & 18,5 \\
\hline & & Chironomus & 6 & 111,1 \\
\hline & & Polypedilum & 2 & 37,0 \\
\hline & Tanypodinae & Monopelia & 1 & 18,5 \\
\hline & Orthocladiinae & Cricotopus & 1 & 18,5 \\
\hline \multicolumn{5}{|l|}{ IG-10 } \\
\hline \multirow{2}{*}{ IG-11 } & \multirow{2}{*}{ Chironominae } & Chironomus & 1 & 18,5 \\
\hline & & Oukuriella & 1 & 18,5 \\
\hline IG-12 & Chironominae & Polypedilum & 2 & 37,0 \\
\hline IG-13 & Chironominae & Polypedilum & 4 & 74,1 \\
\hline \multicolumn{5}{|l|}{ IG-14 } \\
\hline \multirow{8}{*}{ IG-15 } & \multirow{5}{*}{ Chironominae } & Polypedilum & 60 & 1111,1 \\
\hline & & Pelomus & 1 & 18,5 \\
\hline & & Tanytarsus & 1 & 18,5 \\
\hline & & Fissimentum & 3 & 55,6 \\
\hline & & Caladomyia & 10 & 18,51 \\
\hline & \multirow{2}{*}{$\begin{array}{l}\text { Complexo } \\
\text { Harnischia }\end{array}$} & Cladopelma & 3 & 55,6 \\
\hline & & Cryptochironomus & 2 & 37,0 \\
\hline & Tanypodinae & Larsia & 1 & 18,5 \\
\hline \multirow{11}{*}{ IG-16 } & \multirow{5}{*}{ Chironominae } & Fissimentum & 4 & 74,0 \\
\hline & & Polypedilum & 246 & 4555,5 \\
\hline & & Caladomyia & 7 & 129,6 \\
\hline & & Psendochirnomus & 2 & 37,0 \\
\hline & & Tanytarsus & 1 & 18,5 \\
\hline & $\begin{array}{l}\text { Complexo } \\
\text { Harnischia }\end{array}$ & Crypotochironomus & 2 & 37,0 \\
\hline & \multirow{3}{*}{ Tanypodinae } & Djalmabatista & 12 & 222,2 \\
\hline & & Larsia & 1 & 18,5 \\
\hline & & Monopelopia & 1 & 18,5 \\
\hline & \multirow{2}{*}{ Orthocladiinae } & Lopescardius & 1 & 18,5 \\
\hline & & Corynoneura & 1 & 18,5 \\
\hline
\end{tabular}


A família Chironomidae e Oligochaeta foram os organismos mais frequentes no rio Iguaçu (Tabela 2). Chironomidae pertence à Ordem Diptera e subordem Nematocera. Trata-se de uma família de mosquitos que coloniza ambientes aquáticos continentais. Aliado a Oligochaeta são os macroinvertebrados bentônicos mais utilizados como bioindicadores, devido a sua elevada riqueza e abundância. Sua escolha deve-se principalmente por sua ampla distribuição ocupando todos os tipos de ambientes aquáticos (HARRISON, 2002; PORINCHU; MACDONALD, 2003), e também pelo fato de tolerarem grande variação na qualidade da água, sendo muito utilizado em biomonitoramento (ADRIAENSSENS et al., 2004; BHATTACHARYAY et al., 2006; JANSSENS DE BISTHOVEN; GERHARDT, 2003; JANSSENS DE BISTHOVEN; GERHARDT; SOARES, 2005, LUOTO, 2011; MOUSAVI; PRIMICERIO; AMUNDSEN, 2003; ).

Alterações na estrutura da comunidade de Chironomidae podem indicar introdução de efluentes industriais, esgotos domésticos e degradação do ecossistema aquático (LUOTO, 2011). Pesquisadores observaram relações entre contaminação ambiental e presença de táxons de Chironomidae. Mousavi, Primicerio e Amundsen (2003), avaliaram a estrutura e diversidade da comunidade ao longo de um gradiente de contaminação por metais pesados. Adriaenssens et al. (2004), verificaram que há gêneros que são sensíveis a alterações na qualidade da água podendo ser divididos em três classes que incluem: indicadores de boa qualidade da água, indicadores de água enriquecida com nutrientes e enriquecida com matéria orgânica.

Esta família também foi foco de estudo em áreas contaminadas por ácido arsênico e contaminação pela água de drenagem de mineração (JANSSENS DE BISTHOVEN; GERHARDT; SOARES, 2005). Também foram observadas alterações morfológicas nos Chironomidae devido a contaminações no ambiente (MAC DONALD; TAYLOR, 2006; NAZAROVA et al., 2004; OCHIENG; DE RUYTER VAN STEVENINCK; WANDA, 2008). A tabela 3 apresenta a densidade e os gêneros de Chironomidae identificados. É possível observar que avaliando a família Chironomidae ao nível de gênero houve variação entre os pontos amostrados, a variação ocorreu principalmente entre o número de gêneros observados em cada ponto, apesar de nos pontos IG-10 e IG-14 não houve a sua ocorrência, também ocorreu variação de densidade entre gêneros e pontos.

Os gêneros Chironomus e Polypedilum foram registrados nos pontos IG-1 a IG-7, alternando-se ou sendo encontrados mesmo ponto, ambos os gêneros são característicos de ambientes ricos em matéria orgânica e baixa concentração de oxigênio dissolvido (CALLISTO; MORETTI; GOULART, 2001; DORNFELD; ESPINDOLA; LEITE, 2005), apesar das condições favoráveis para uma colonização com sucesso devido a disponibilidade de alimento, foram observados baixas densidades. Estes organismos possuem hemoglobina, o que faz com que armazenem maior quantidade de oxigênio possibilitando habitarem ambientes com anoxia ou baixa concentração de oxigênio dissolvido. Este trecho do rio Iguaçu encontra-se inserido na RMC e tem como principal fator de degradação da qualidade da água a introdução de esgotos domésticos in natura (KNAPIK; FERNANDES; AZEVEDO, 2011).

A partir do IG-8 gradualmente foi registrado maior número de gêneros de Chironomidae, o que indica que a qualidade da água também apresentou melhora. No ponto IG-8, além da presença de Chironomus e Polypedilum, também foi observado Thienemanniella (subfamília Orthocladiinae), considerada sensível a depleção de oxigênio dissolvido. Sua presença foi observada em novembro de 2012 onde a concentração de OD foi de 6,07 $\mathrm{mg} / \mathrm{L}$. Apesar de ser observado um táxon sensível, a densidade do Polypedilum foi muito superior, $2370,4 \mathrm{ind} / \mathrm{m}^{2}$, ao contrário dos outros gêneros, 18,5 para Chironomus e 129,6 ind $/ \mathrm{m}^{2}$ para Thienemanniella, um indicativo da presença de matéria orgânica embora em concentrações menores.

Cricotopus foi registrado no ponto IG-9, este gênero é normalmente associado a riachos de altitude e com boa qualidade da água. A influência da introdução de esgotos a partir de uma estação de tratamento de esgotos e do município de Porto Vitória foi observada sobre a comunidade bentônica, nos pontos IG-12 e IG-13, onde foi observado somente o gênero Polypedilum. Nos pontos IG-15 e IG-16 foi observada elevada riqueza de táxons, nestes pontos o rio possui boa qualidade da água e parte de seu percurso está inserido no Parque Nacional do Iguaçu.

O IG-15 apresentou 8 táxons diferentes, como ocorreu no IG-8, o gênero Polypedilum teve a maior densidade (1111,1 ind/m2). No ponto IG-16 a diversidade de táxons foi maior do que no IG-15, total de 12 táxons, sendo que a densidade de Polypedilum apresentou o maior valor entre todos os pontos monitorados, 4555,5 ind $/ \mathrm{m}^{2}$. Embora as densidades de Polypedilum tenham sido elevadas deve ser destacado que sua presença é um indicador de contaminação por matéria orgânica quando a presença deste gênero é restrita ou em conjunto com outro organismo tolerante. Quando encontra-se com vários outros gêneros de Chironomidae, incluindo táxons sensíveis, elevadas densidades podem ser observadas por ser um organismo cosmopolita, colonizando qualquer tipo de ambiente com sucesso.

Os resultados dos gêneros de Chironomidae diferenciaram entre um ambiente com integridade ecológica em relação aos trechos onde há ocupação urbana ou agrícola. Resultados semelhantes foram encontrados por Kafle et al. (2013), Luoto (2011) e Odume e Mueller (2011). Em relação aos resultados de vazão (Tabela 4), a maior riqueza de Chironomidae foi registrada nos pontos onde houve maior variação da vazão e melhor qualidade da água. O escoamento superficial pode carrear galhos, folhas e matéria orgânica, disponibilizando a criação e novos habitats para colonização por meio da introdução de material alóctone (CALLISTO; MORETTTI; GOULART, 2001). A partir do ponto IG-10 o rio Iguaçu apresenta um grande aumento em sua vazão devido a entrada de muitos afluentes como o rio Negrinho, rio da Várzea e rio Potinga.

\section{QUALIDADE DA ÁGUA}

$\mathrm{Na}$ tabela 4 constam os valores médios e desvio padrão dos parâmetros quantificados. De acordo com os resultados foi possível distinguir duas tendências em relação a qualidade da água assim como ocorreu com a fauna bentônica em relação ao gêneros de Chironomidae, a primeira de ambiente impactado na RMC, e a segunda a partir do IG-9 a melhora da qualidade 
Tabela 4 - Caracterização física e química da água do rio Iguaçu e vazões medidas (média \pm desvio padrão)

\begin{tabular}{|c|c|c|c|c|c|c|c|c|c|c|c|}
\hline Pontos & $\begin{array}{l}\text { STD } \\
(\mathrm{mg} / \mathrm{L})\end{array}$ & $\begin{array}{l}\text { Condutividade } \\
\left(\mu \mathrm{s} / \mathrm{cm}^{2}\right)\end{array}$ & $\mathrm{pH}$ & $\begin{array}{c}\mathrm{OD} \\
(\mathrm{mg} / \mathrm{L})\end{array}$ & $\begin{array}{c}\text { Nitrogênio } \\
\text { Amoniacal } \\
(\mathrm{mg} / \mathrm{L})\end{array}$ & $\begin{array}{l}\text { Nitrato } \\
(\mathrm{mg} / \mathrm{L})\end{array}$ & $\begin{array}{l}\text { Ortofosfato } \\
(\mathrm{mg} / \mathrm{L})\end{array}$ & $\begin{array}{c}\text { PT } \\
(\mathrm{mg} / \mathrm{L})\end{array}$ & $\begin{array}{l}\text { COD } \\
(\mathrm{mg} / \mathrm{L})\end{array}$ & $\begin{array}{l}\text { Vazão } \\
\left(\mathrm{m}^{3} / \mathrm{s}\right)\end{array}$ & $\mathrm{n}^{*}$ \\
\hline IG-1 & $\begin{array}{c}169,50 \\
\pm \\
54,36\end{array}$ & $\begin{array}{c}252,15 \\
\pm \\
166,55\end{array}$ & $\begin{array}{c}6,93 \\
\pm \\
0,13\end{array}$ & $\begin{array}{c}3,34 \\
\pm \\
1,33\end{array}$ & $\begin{array}{c}11,59 \\
\pm \\
4,58\end{array}$ & $\begin{array}{c}1,57 \\
\pm \\
1,49\end{array}$ & $\begin{array}{c}0,41 \\
\pm \\
0,27\end{array}$ & $\begin{array}{c}0,84 \\
\pm \\
0,94\end{array}$ & $\begin{array}{c}15,99 \\
\pm \\
11,02\end{array}$ & $\begin{array}{c}6,74 \\
\pm \\
4,21\end{array}$ & 4 \\
\hline IG-2 & $\begin{array}{c}206,25 \\
\pm \\
43,44 \\
\end{array}$ & $\begin{array}{c}285,43 \\
\pm \\
181,55\end{array}$ & $\begin{array}{c}7,50 \\
\pm \\
0,83\end{array}$ & $\begin{array}{c}0,80 \\
\pm \\
1,51\end{array}$ & $\begin{array}{c}14,41 \\
\pm \\
578\end{array}$ & $\begin{array}{c}2,29 \\
\pm \\
3,44\end{array}$ & $\begin{array}{c}0,54 \\
\pm \\
0,31\end{array}$ & $\begin{array}{c}0,90 \\
\pm \\
0,57\end{array}$ & $\begin{array}{c}19,61 \\
\pm \\
14,06\end{array}$ & - & 4 \\
\hline IG-3 & $\begin{array}{c}124,00 \\
\pm \\
69,72\end{array}$ & $\begin{array}{c}216,00 \\
\pm \\
113,25\end{array}$ & $\begin{array}{c}7,73 \\
\pm \\
0,29\end{array}$ & $\begin{array}{c}1,30 \\
\pm \\
0,37\end{array}$ & $\begin{array}{c}8,62 \\
\pm \\
5,24\end{array}$ & $\begin{array}{c}1,00 \\
\pm \\
1,06\end{array}$ & $\begin{array}{c}0,53 \\
\pm \\
0,34\end{array}$ & $\begin{array}{c}0,64 \\
\pm \\
0,44\end{array}$ & $\begin{array}{c}14,40 \\
\pm \\
8,29\end{array}$ & $\begin{array}{c}20,16 \\
\pm \\
11,01\end{array}$ & 4 \\
\hline IG-4 & $\begin{array}{c}1111,75 \\
\pm \\
69,72 \\
\end{array}$ & $\begin{array}{c}192,50 \\
\pm \\
108,76 \\
\end{array}$ & $\begin{array}{c}7,52 \\
\pm \\
0,68 \\
\end{array}$ & $\begin{array}{c}1,00 \\
\pm \\
0,77 \\
\end{array}$ & $\begin{array}{c}8,18 \\
\pm \\
6,82 \\
\end{array}$ & $\begin{array}{c}0,75 \\
\pm \\
0,78 \\
\end{array}$ & $\begin{array}{c}0,51 \\
\pm \\
0,34\end{array}$ & $\begin{array}{c}0,42 \\
\pm \\
0,24 \\
\end{array}$ & $\begin{array}{c}12,86 \\
\pm \\
5,42 \\
\end{array}$ & - & 4 \\
\hline IG-5 & $\begin{array}{c}124,50 \\
\pm \\
70,50\end{array}$ & $\begin{array}{c}217,50 \\
\pm \\
117,56\end{array}$ & $\begin{array}{c}7,42 \\
\pm \\
0,29\end{array}$ & $\begin{array}{c}0,79 \\
\pm \\
1,19\end{array}$ & $\begin{array}{c}8,49 \\
\pm \\
5,42\end{array}$ & $\begin{array}{c}0,92 \\
\pm \\
0,82\end{array}$ & $\begin{array}{c}0,43 \\
\pm \\
0,26\end{array}$ & $\begin{array}{c}0,44 \\
\pm \\
0,37\end{array}$ & $\begin{array}{c}12,85 \\
\pm \\
4,54\end{array}$ & & 4 \\
\hline IG-6 & $\begin{array}{c}119,25 \\
\pm \\
58,66\end{array}$ & $\begin{array}{c}189,00 \\
\pm \\
109,12\end{array}$ & $\begin{array}{c}7,21 \\
\pm \\
0,24\end{array}$ & $\begin{array}{c}0,73 \\
\pm \\
1,00\end{array}$ & $\begin{array}{c}5,51 \\
\pm \\
4,70\end{array}$ & $\begin{array}{c}0,88 \\
\pm \\
0,99\end{array}$ & $\begin{array}{c}0,59 \\
\pm \\
0,60\end{array}$ & $\begin{array}{c}0,67 \\
\pm \\
0,81\end{array}$ & $\begin{array}{c}12,04 \\
\pm \\
4,55\end{array}$ & $\begin{array}{c}61,45 \\
\pm \\
34,75\end{array}$ & 4 \\
\hline IG-7 & $\begin{array}{c}103,00 \\
\pm \\
45,88\end{array}$ & $\begin{array}{c}184,00 \\
\pm \\
85,25\end{array}$ & $\begin{array}{c}7,43 \\
\pm \\
0,29\end{array}$ & $\begin{array}{c}1,80 \\
\pm \\
1,83\end{array}$ & $\begin{array}{c}4,97 \\
\pm \\
2,71\end{array}$ & $\begin{array}{c}0,83 \\
\pm \\
0,58\end{array}$ & $\begin{array}{c}0,30 \\
\pm \\
0,21\end{array}$ & $\begin{array}{c}0,36 \\
\pm \\
0,33\end{array}$ & $\begin{array}{c}11,89 \\
\pm \\
3,85\end{array}$ & $\begin{array}{c}68,73 \\
\pm \\
32,24\end{array}$ & 4 \\
\hline IG-8 & $\begin{array}{c}71,50 \\
\pm \\
31,08\end{array}$ & $\begin{array}{c}132,50 \\
\pm \\
64,40\end{array}$ & $\begin{array}{c}8,00 \\
\pm \\
0,27\end{array}$ & $\begin{array}{c}6,84 \\
\pm \\
1,01\end{array}$ & $\begin{array}{c}2,45 \\
\pm \\
1,45\end{array}$ & $\begin{array}{c}3,14 \\
\pm \\
3,15\end{array}$ & $\begin{array}{c}0,17 \\
\pm \\
0,09\end{array}$ & $\begin{array}{c}0,18 \\
\pm \\
0,05\end{array}$ & $\begin{array}{c}7,81 \\
\pm \\
2,08\end{array}$ & $\begin{array}{c}73,61 \\
\pm \\
26,76\end{array}$ & 4 \\
\hline IG-9 & $\begin{array}{c}42,00 \\
\pm \\
19,11\end{array}$ & $\begin{array}{c}76,25 \\
\pm \\
36,84\end{array}$ & $\begin{array}{c}7,60 \\
\pm \\
0,58\end{array}$ & $\begin{array}{c}5,41 \\
\pm \\
1,84\end{array}$ & $\begin{array}{c}0,29 \\
\pm \\
0,19\end{array}$ & $\begin{array}{c}2,07 \\
\pm \\
0,91\end{array}$ & $\begin{array}{c}0,07 \\
\pm \\
0,05\end{array}$ & $\begin{array}{c}0,17 \\
\pm \\
0,12\end{array}$ & $\begin{array}{c}5,51 \\
\pm \\
1,81\end{array}$ & $\begin{array}{c}128,25 \\
\pm \\
64,81\end{array}$ & 4 \\
\hline IG-10 & $\begin{array}{c}15,33 \\
\pm \\
7,64\end{array}$ & $\begin{array}{c}29,00 \\
\pm \\
17,06\end{array}$ & $\begin{array}{c}7,87 \\
\pm \\
0,70\end{array}$ & $\begin{array}{c}6,72 \\
\pm \\
2,28\end{array}$ & $\begin{array}{c}0,10 \\
\pm \\
0,06\end{array}$ & $\begin{array}{c}1,27 \\
\pm \\
0,63\end{array}$ & $\begin{array}{c}0,06 \\
\pm \\
0,05\end{array}$ & $\begin{array}{c}0,16 \\
\pm \\
0,07\end{array}$ & $\begin{array}{c}4,59 \\
\pm \\
2,65\end{array}$ & $\begin{array}{c}684,05 \\
\pm \\
370,12\end{array}$ & 4 \\
\hline IG-11 & $\begin{array}{c}13,00 \\
\pm \\
9,90\end{array}$ & $\begin{array}{c}27,33 \\
\pm \\
16,26\end{array}$ & $\begin{array}{c}7,72 \\
\pm \\
0,51\end{array}$ & $\begin{array}{c}6,43 \\
\pm \\
1,63\end{array}$ & $\begin{array}{c}0,16 \\
\pm \\
0,08\end{array}$ & $\begin{array}{c}1,32 \\
\pm \\
0,63\end{array}$ & $\begin{array}{c}0,05 \\
\pm \\
0,04\end{array}$ & $\begin{array}{c}0,16 \\
\pm \\
0,14\end{array}$ & $\begin{array}{c}13,99 \\
\pm \\
10,65\end{array}$ & $\begin{array}{c}684,05 \\
\pm \\
370,12\end{array}$ & 4 \\
\hline IG-12 & $\begin{array}{c}16,00 \\
\pm \\
8,89\end{array}$ & $\begin{array}{c}30,33 \\
\pm \\
18,72\end{array}$ & $\begin{array}{c}7,84 \\
\pm \\
1,36\end{array}$ & $\begin{array}{c}6,92 \\
\pm \\
1,68\end{array}$ & $\begin{array}{c}0,16 \\
\pm \\
0,09\end{array}$ & $\begin{array}{c}1,32 \\
\pm \\
0,60\end{array}$ & $\begin{array}{c}0,05 \\
\pm \\
0,04\end{array}$ & $\begin{array}{c}0,17 \\
\pm \\
0,18\end{array}$ & $\begin{array}{c}5,93 \\
\pm \\
2,73\end{array}$ & $\begin{array}{c}684,05 \\
\pm \\
370,12\end{array}$ & 4 \\
\hline IG-13 & $\begin{array}{c}13,20 \\
\pm \\
13,24\end{array}$ & $\begin{array}{c}27,67 \\
\pm \\
22,74\end{array}$ & $\begin{array}{c}7,90 \\
\pm \\
1,03\end{array}$ & $\begin{array}{c}6,20 \\
\pm \\
1,59\end{array}$ & $\begin{array}{c}0,11 \\
\pm \\
0,05\end{array}$ & $\begin{array}{c}1,17 \\
\pm \\
0,54\end{array}$ & $\begin{array}{c}0,07 \\
\pm \\
0,06\end{array}$ & $\begin{array}{c}0,13 \\
\pm \\
0,10\end{array}$ & $\begin{array}{c}7,70 \\
\pm \\
6,57\end{array}$ & $\begin{array}{c}833,28 \\
\pm \\
535,52\end{array}$ & 3 \\
\hline IG-14 & $\begin{array}{c}5,00 \\
\pm \\
3,61 \\
\end{array}$ & $\begin{array}{c}9,67 \\
\pm \\
7,64 \\
\end{array}$ & $\begin{array}{c}7,21 \\
\pm \\
0,17 \\
\end{array}$ & $\begin{array}{c}6,13 \\
\pm \\
4,10 \\
\end{array}$ & $\begin{array}{c}0,02 \\
\pm \\
0,04\end{array}$ & $\begin{array}{c}0,57 \\
\pm \\
0,47 \\
\end{array}$ & $\begin{array}{c}0,00 \\
\pm \\
0,01 \\
\end{array}$ & $\begin{array}{c}0,10 \\
\pm \\
0,07 \\
\end{array}$ & $\begin{array}{c}5,39 \\
\pm \\
2,91 \\
\end{array}$ & 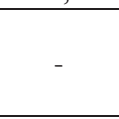 & 3 \\
\hline IG-15 & $\begin{array}{c}5,33 \\
\pm \\
3,06\end{array}$ & $\begin{array}{c}10,33 \\
\pm \\
7,02 \\
\end{array}$ & $\begin{array}{c}7,48 \\
\pm \\
0,21\end{array}$ & $\begin{array}{c}6,51 \\
\pm \\
1,35\end{array}$ & $\begin{array}{c}0,03 \\
\pm \\
0,05\end{array}$ & $\begin{array}{c}0,59 \\
\pm \\
0,49\end{array}$ & $\begin{array}{c}0,00 \\
\pm \\
0,00\end{array}$ & $\begin{array}{c}0,22 \\
\pm \\
0,30\end{array}$ & $\begin{array}{c}5,23 \\
\pm \\
3,93\end{array}$ & - & 3 \\
\hline IG-16 & $\begin{array}{c}5,33 \\
\pm \\
4,04\end{array}$ & $\begin{array}{c}10,33 \\
\pm \\
7,64\end{array}$ & $\begin{array}{c}8,18 \\
\pm \\
0,94\end{array}$ & $\begin{array}{c}9,15 \\
\pm \\
0,88\end{array}$ & $\begin{array}{c}0,04 \\
\pm \\
0,07\end{array}$ & $\begin{array}{c}0,64 \\
\pm \\
0,65\end{array}$ & $\begin{array}{c}0,00 \\
\pm \\
0,00\end{array}$ & $\begin{array}{c}0,19 \\
\pm \\
0,202\end{array}$ & $\begin{array}{c}3,15 \\
\pm \\
2,54\end{array}$ & $\begin{array}{c}2847,09 \\
\pm \\
305,07\end{array}$ & 3 \\
\hline
\end{tabular}

*Número de coletas realizadas

da água em função do uso e ocupação do solo (predominância de atividade agrícola e centros urbanos pequenos).

As concentrações de sólidos totais dissolvidos (STD) acompanharam o gradiente de poluição do rio, sendo que nos pontos IG-1 a IG-8 foi observada elevadas concentrações (230,00 a 111,00 mg/L, valores máximos). A partir do IG-9 o rio apresentou valores inferiores $(70,00 \mathrm{mg} / \mathrm{L})$. Os valores de condutividade, parâmetro que pode indicar a contaminação por esgotos domésticos apresentaram tendência similar ao de STD.
A resolução CONAMA 357/2005 estabelece valores máximos e mínimos de pH entre 6,0 a 9,0 para ambientes lóticos Classe 2. No rio Iguaçu todos os valores estiveram dentro desses limites. Os resultados das concentrações de oxigênio dissolvido indicaram o real o impacto na qualidade da água causado pela introdução de esgotos in natura ou da baixa cobertura de rede de esgoto e estações de tratamento. Entre os pontos IG-1 a IG-7 as concentrações permaneceram inferiores a $5 \mathrm{mg} / \mathrm{L}$, o qual é o limite inferior para rio de classe 2 , de acordo com a 
Bem et al.: Qualidade da água de um ambiente lótico sob impacto antropogênico e sua comunidade bentônica

Tabela 5 - Resultado da análise de regressão múltipla da fauna de Chironomidae e os parâmetros mensurados

\begin{tabular}{c|c|c|c}
\hline Variáveis resposta & Equação & $\mathbf{R}^{2}$ & \multicolumn{1}{c}{0,44} \\
\hline Polypedilum & Densidade = Cond.x0,295+Nit.x0,393+Qx0,732 & 0,0220 \\
\hline $\begin{array}{c}\text { Thienamanniella } \\
\begin{array}{c}\text { Carsia, Tanytarsus, Fissimentum, } \\
\text { Pseudochironomus, Corynoneura, } \\
\text { Djalmabatista, Lopescardius }\end{array}\end{array}$ & Densidade = Odx0,280+Condx0,862+Nitx0,725- & 0,47 \\
\hline STDx0,630 & Densidade $=$ Qx0,614 & 0,38 \\
\hline
\end{tabular}

Legenda: Cond.: Condutividade, Nit.: Nitrato, Q: vazão, OD: Oxigênio dissolvido, STD: sólidos totais dissolvidos

resolução 357/2005. O ponto IG-8, localizado no município de Porto Amazonas, apresentou o ponto do início da melhora da qualidade da água em relação à concentração de oxigênio dissolvido (8,31 mg/L, valor máximo), principalmente, devido à capacidade de autodepuração do rio no seu percurso, aumento da vazão e diminuição da contribuição por lançamentos pontuais.

Além da baixa concentração de OD, também entre os pontos IG-1 a IG-7 as concentrações de nitrogênio amoniacal foram elevadas $(18,45 \mathrm{mg} / \mathrm{L}$ a 8,02 , valores máximos) e de nitrato baixas $(3,76$ a $1,68 \mathrm{mg} / \mathrm{L}$, valores máximos) respectivamente, evidenciando a introdução de esgotos domésticos. Entre os pontos IG-1 e IG-8 foram encontrados valores elevados da concentração de ortofosfato $(0,81 \mathrm{mg} / \mathrm{L}$ a $0,29 \mathrm{mg} / \mathrm{L}$, valores máximos) em comparação aos pontos a montante do IG-9 $(0,13 \mathrm{mg} / \mathrm{L})$. As concentrações de fósforo total (PT) foram igualmente elevadas principalmente nos pontos presentes na RMC (1,93 a 0,74 mg/L, valores máximos), concentrações superiores a 0,1 mg/L estabelecido pelo CONAMA 357/05.

As concentrações das formas dissolvidas de carbono contemplam a matéria orgânica dissolvida em todos os estados de oxidação. Em rios poluídos os valores das concentrações de COD podem ser elevados, em parte o aumento da concentração desta forma de carbono esta relacionada com a introdução de matéria orgânica por esgotos domésticos lançados in natura. Os valores observados entre os pontos IG-1 a IG8 foram elevados (28,20 a $14,77 \mathrm{mg} / \mathrm{L}$, valores máximos) corroborando com os resultados dos demais parâmetros, somente no ponto IG11 houve aumento da concentração, provavelmente devido a lançamentos de esgotos domésticos sem tratamento, no ponto IG-12 encontra-se uma estação de tratamento de esgoto.

A RLM indicou que os gêneros de Chironomidae Chironomus, Axarus, Oukuriella, Cladopelma, Monopelopia e Pelomus não apresentaram relação com os parâmetros analisados. Entretanto, os gêneros Polypedilum, Thienemaniella, Larsia, Tanytarsus, Fissimentum, Caladomya, Pseudochironomus, Corynoneura, Djalmabatista e Lopescardius (Tabela 5) apresentaram. De acordo com a RLM o gênero Polypedilum foi relacionado positivamente com a condutividade, nitrato e vazão, apresentando sua afinidade com a matéria orgânica. Thienemanniella relacionou-se com positivamente com condutividade, oxigênio dissolvido e nitrato e negativamente com sólidos totais dissolvidos, gênero sensível a ambientes impactados e exigente em relação a concentração de oxigênio dissolvido. Os demais gêneros relacionaram-se positivamente com a vazão, a vazão mostrou-se como uma importante variável, é responsável pelo estabelecimento da comunidade e por criação de novos hábitats e segundo Arthington et al. (2010), as variáveis físico-químicas não surgem da vazão da água, mas sim são influenciadas por ela.

\section{CONCLUSÕES}

Os dados de qualidade da água indicaram que entre os pontos IG-1 a IG-8, o rio Iguaçu apresentou degradação na sua qualidade devido ao lançamento de esgotos pela RMC. Os dados de macroinvertebrados bentônicos em nível de grupos não possibilitaram observar mudanças na estrutura da comunidade suficientes para inferir características qualitativas referentes a qualidade da água, somente os táxons Nematode e Hydrachnidae foram restritos entre os pontos pertencentes a RMC.

Por outro lado, a avaliação das larvas de Chironomidae ao nível de gênero foi interessante e relevante para a gestão de recursos hídricos. Entre os pontos IG-1 e IG-7 foram registrado somente os gêneros Chironomus e Polypedilum, os quais são organismos resistentes e capazes de colonizar ambientes ricos em matéria orgânica e baixa concentração de oxigênio dissolvido, portanto, são os gêneros registrados mais sensíveis para indicar esta forma de alteração de qualidade.

A partir do ponto IG-8 foi registrado gradualmente maior riqueza de gêneros, inclusive alguns táxons sensíveis (Orthocladiinae), indicando que estes ambientes possuem boa qualidade da água e não sofrem impactos significantes referentes a fontes pontuais ou difusas.

Apesar da reposta coerente dos gêneros de Chironomidae com os dados de qualidade da água, os resultados da análise de regressão linear múltipla indicaram que a vazão foi uma importante variável, responsável pelo estabelecimento da comunidade, provavelmente devido a criação de novos hábitats e pelo efeito de concentração, diluição e aporte de poluentes.

Além da indicação da qualidade do ambiente não em termos de valores como ocorre com parâmetros físico-químicos, mas também em termos de uma qualidade biológica, os macroinvertebrados bentônicos podem indicar a qualidade da água de forma temporal, isto é, indicar variações na qualidade que podem ocorrer entre as coletas. Resultado que possui grande significado para a gestão, pois em geral os rios são amostrados com uma frequência bimestral ou trimestral.

Ademais aos parâmetros tradicionais de qualidade da água, o uso de macroinvertebrados bentônicos pode colaborar para indicar outras substâncias que eventualmente prejudiquem 
a fauna e não estão sendo consideradas no monitoramento.

\section{AGRADECIMENTOS}

Os autores agradecem ao CNPq, CAPES e Fundação Araucária pelo apoio financeiro.

\section{REFERÊNCIAS}

ADRIAENSSENS, V.; SIMONS, F.; NGUYEN, L. T. H.; GODDEERIS, B.; GOETHALS, P. L. M. DEPAUW, N. Potential of bio-indication of chironomid communities for assessment of running water quality in Flanders (Belgium). Belgian Journal of Zoology, v. 134, n. 1, p. 32-40, 2004.

APHA. Standard Methods for the Examination of Water and Wastewater. 20th ed. Washington: APHA, 1998.

ARTHINGTON, A. H.; NAIMAN, R. J.; McCLAIN, M. E.; NILSSON, C. Preserving the biodiversity and ecological services of rivers and research opportunities. Freshwater Biology, v. 55, n. 1, p. 1-16, Jan. 2010.

BHATTACHARYAY, G.; SADHU, A. M.; MAJUMDAR, U.; CHAUDHURI, P. K.; ALI, A. Assessment of impact of heavy metals on the communities and morphological deformities of Chironomidae lavae in the River Damodar (India, West Bengal). Supplementa ad Acta Hydrobiologica, v. 8, p. 21-32, June 2006.

BRACCIA, A.; VOSHELL-JR, J. R. Environmental factors accounting for benthic macroinvertebrate assemblage structure at the sample scale in streams subjected to a gradient of cattle grazing. Hydrobiologia, v. 573, n. 1, p. 55-73, Dec. 2006.

BRASILa. Constituição da República Federativa do Brasil. Diário Oficial da Republica Federativa do Brasil, Brasília, DF, 2013. Disponível em: <http://www.planalto.gov.br/ccivil_03/constituicao/ constituicaocompilado.htm>. Acesso em: 7 jan. 2013.

BRASILb. Resolução CONAMA 357, 17 de março de 2005. Diário Oficial da Republica Federativa do Brasil, Brasília, DF, 2006. Disponível em: <http://www.mma.gov.br/conama>. Acesso em: 12 fev. 2006.

BUSS, D. F.; BAPTISTA, D. F.; NESSIMIAN, J. L.; EGLER, M. E. Substrate specicity, environmental degradation and disturbance structuring macroinvertebate assemblages in neotropical streams. Hidrobiologia, v. 518, n. 1-3, p. 179-188, May 2004.

BUSS, D. F.; BAPTISTA, D. F.; SILVEIRA, M. P.; NESSIMIAN, J. L.; DORVILLÉ, L. F. Influence of water chemistry and environmental degradation on macroinvertebrate assemblages in a river basin in south-east Brazil. Hydrobiologia, v. 481, n. 1-3, p. 125-136, Aug. 2002.
CAllisto, M.; MORETTI, M.; GOUlART, M. Macroinvertebrados bentônicos como ferramenta para avaliar a saúde de riacho. Revista Brasileira de Recursos Hídricos, v. 6, n. 1, p. 71-82, jan./mar. 2001.

DOMINGUES, D. D.; STAHNKE, L. F.; FIORENTIN, L. G.; HANSEN, M. A. F. Análise hidroquímica, metais pesados em sedimentos e de macroinvertebrados aquáticos no Arroio da Manteiga, são Leopoldo, RS. Revista Brasileira de Recursos Hídricos, v. 12, n. 1, p. 233-243, jan./mar. 2007.

DORNFELD, C. B.; ESPINDOLA, E. L. G.; LEITE, M. A. Avaliação da eutrofização e sua relação com Chironomidae no rio Atibaia e reservatório de Salto Grande (Americana, SPBrasil). Revista Brasileira de Recursos Hídricos, v. 10, n. 3, p. 53-62, jul./set. 2005.

FERREIRA, W. R.; RODRIGUES, D. N.; ALVES, C. B. M.; CALLISTO, M. Biomonitoramento de longo prazo da Bacia do Rio das Velhas através de um índice multimétrico bentônico. Revista Brasileira de Recursos Hídricos, v. 17, n. 3, p. 253-259, jul./ set. 2012.

HARE, L. Aquatic insects and trace metals: bioavailability, bioaccumulation and toxicology. Critical Reviews in Toxicology, v. 22, n. 5-6, p. 327-369, 1992.

HARE, L.; CAMPBELL, P. G. C. Temporal variations of trace metals in aquatic insects. Freshwater Biology, v. 27, n. 1, p. 13-2, Feb. 1992

HARRIS, J. A.; HOBBS, R. J.; HIGGS, E.; AROSON, J. Ecological restoration and global climate changes. Restoration Ecology, v. 14, n. 2 , p. $170-176$, June 2006

HARRISON, A. D. Guides to the Freshwater Invertebrates of Southern Africa. South Africa: Water Research Comission, 2002.

HEPP, L. U.; SANTOS, S. Benthic communities of streams related to different land uses in a hydrographic basin in southern Brazil. Environmental Monitoring and Assessment, v. 157,n. 1-4, p. 305-318, Oct. 2009.

JANSSENS DE BISTHOVEN, L.; GERHARDT, A. Chironomidae (Diptera, Nematocera) fauna in three small streams of Skania, Sweden. Environmental monitoring and Assessment, v. 83, n. 1, p. 89-102, Mar. 2003.

JANSSENS DE BISTHOVEN, L., GERHARDT, A. e SOARES, A. M. V. M. Chironomidae larvae as bioindicators of an acid mine drainage in Portugal. Hydrobiologia, v. 512, n. 1-3, p. $181-$ 191, Jan. 2005.

KAFLE, A.; TROELSTRUP, N. H. Jr.; KRAUSE, J. R.; BERTRAND, K. N. Assemblage structure of Chironomidae (Diptera: Insecta) from wadeable streams of the northern glaciated plains, South Dakota, USA. In: SOUTH DAKOTA 
ACADEMY OF SCIENCE, 2013, South Dakota. Proceedings... [S.1: s.n.], 2013. v. 92, p. 31-49.

KNAPIK, H. G.; FERNANDES, C. V. S.; AZEVEDO, J. C. R. Characterization of organic matter using fluorescence and absorbance spectroscopy: the case study of a Brazilian polluted river. In: IWA SPECIALTY CONFERENCE ON NATURAL ORGANIC MATTER, 4., 2011, Costa Mesa, Califórnia. Proceedings... Califórnia: IWA, 2011.

LUOTO, T. P. The relationships between water quality and chironomid distribution Finland- a new assemblage-based tool for assessments of long-term nutria dynamics. Ecological Indicators, v. 11, p. 255-262, 2011.

LUZ, L. D.; FERREIRA, M. T. A questão ecológica na gestão dos corpos hídricos: analisando os focos das diretrizes brasileira e europeia. Revista de Gestão de Água da América Latina, v. 8, n. 1, p. 19-31, jan./jun. 2011.

LYNCH, T. R.; POPP, C. J.; JACOBI, G. Z. Aquatic insects as environmental monitors of trace metal contamination: Red River, New Mexico. Water, Air, \& Soil Pollution, v. 42, n. 1-2, p. 19-31, Nov. 1988.

MACDONALD, E. E.; TAYLOR, R. Incidence of mentum deformities in midge larvae (Diptera: Chironomidae) from Northern Nova Scotia, Canada. Hydrobiologia, v. 563, n. 1, p. 277-287, June 2006.

MILOSEVIC, D.; STOJKOVIC, M.; CERBA, D.; VLADICA, S. Different aggregation approaches in the chironomid community and threshold of acceptable information loss. Hydrobiologia, v. 727, n. 1, p. 35-50, Apr. 2014.

MOPHIN-KANI, K.; MURUGESAN, A. G. Assessment of River Water Quality Using Macroinvertebrate Organisms as Pollution Indicators of Tamirabarani River Basin, Tamil Nadu, India. International Journal of Environmental Protection, v. 4, n.1, p. 1-14, Jan. 2014.

MORENO, P.; FRANÇA, J. S.; FERREIRA. W., R.; PAZ. A. D.; MONTEIRO, I. M.; CALLISTO, M. Use of the BEAST model for biomonitoring water quality in a neotropical basin. Hydrobiologia, v. 630, n. 1, p. 231-242, Sept. 2009.

MOUSAVI, S. K.; PRIMICERIO, R.; AMUNDSEN, P. Diversity and structure of Chironomidae (Diptera) communities along a gradient of heavy metal contamination in a subarctic water course. Science of the Total Environment, v. 307, n. 1-3, p. 93-110, May 2003.

MYSLINSKI, E.; GINSBURG, W. Macro-invertebrates as indicators of pollution. Journal of the American Water Works Association, v. 69, p. 538-544, 1977.
NAZAROVA, L. B., RISS, H. W., KAHLHEBER, A.;WERDING, B. Some observations of buccal deformities in Chironomid larvae (Diptera: Choronomidae) from the Cienaga Grande de Santa Marta, Columbia. Caldasia, v. 26, n. 1, p. 275-290, June 2004.

NIYOGI, D. K.; KOREN, M.; ARBUCKLE, C. J.;TOWNSEND, C. R. Stream communities along a catchment land-use gradient: Subsidy-stress responses to pastoral development. Environmental Management, v. 39, n. 2, p. 213-225, Feb. 2007.

OCHIENG, H.; DE RUYTER VAN STEVENINCK, E. D.;WANDA, F. M. Mouthpart deformities in Chironomidae (Diptera) as indicators of heavy metal pollution in northern Lake Victoria, Uganda. African Journal of Aquatic Science, v. 33, n. 2, p. 135-142, 2008.

ODUME, O. N.; MUELLER, W. J. Diversity and structure of Chironomidae communities in relation to water quality differences in the Swartkops River. In: WATERNET/WARFSA/GWP-SA SYMPOSIUM: IWRM FOR NATIONAL AND REGIONAL INTEGRATION THROUGH SCIENCE, POLICY AND PRACTICE, 11., 2011, Maputo, Moçambique. Proceedings... Maputo: [s.n.] 2011.

PORINCHU, D. F.;MACDONALD, G. M. The use and application of freshwater midges (Chironomidae: Insecta: Diptera) in geographical research. Progress in Physical Geography, v. 27 , n. 3, p. 378-422, 2003.

REZENDE, R. S.; GONÇALVES, J. F.; PETRUCIO, M. M. Leaf breakdown and invertebrate colonization of Eucalyptus grandis (Myrtaceae) and Hirtella glandulosa (Chrysobalanaceae) in two Neotropical lakes. Acta Limnologica Brasiliensia, v. 22, n. 1, p. 23-34, 2010.

ROSENBERG, D. M.; WIENS, A. P. Community and species responses of Chironomidae (Diptera) to contamination of freshwaters by crude oil and petroleum products, with special reference to the trail river, Northwest Territories. Journal of the Fisheries Research Board of Canada, v. 33, n. 9, p. 1955-1963, 1976.

SANTOS, L. H. M. L. M.; ARAÚJO, A. N.; FACHINI, A.; PENA, A.; DELERUE-MATOS C.; MONTENEGRO, M. C. B. S. M. Ecotoxicological aspects related to the presence of pharmaceuticals in the aquatic environment. Journal of Hazardous Materials, v. 175, n. 1-3, p. 45-95, Mar. 2010.

SILVA, S. Estudo das larvas de geo-helmintos, in vivo, como bioindicadora de poluição ambiental, em amostras de águas residuárias da ETE contorno de Feira de Santana-Babia: estudo comparativo com diagnóstico de estrongiloidíase. 2009. Monografia - Universidade Estadual de Feira de Santana, Feira de Santana, 2009.

SILVEIRA, M. P.; BUSS, D. F.; NESSIMIAN, J. L.; BAPTISTA, D. F. Spatial and temporal distribution of benthic macroinvertebrates in a southeastern Brazilian river. Brazilian Journal of Biology, v. 66, n. 2B, p. 623-632, May 2006. 
STEWART, P. M.; BUTCHER, J. T.;SWINFORD, T. O. Land use, habitat and water quality effects on macroinvertebrate communities in three watersheds of a Lake Michigan associated marsh system. Aquatic Ecosystem Health \& Management, v. 3, n. 1, p. 179-189, 2000.

TRIVINHO-STRIXINO, S. Larvas de Chironomidae: guia de identificação. São Carlos: Depto Hidrobiologia/Lab. Entomologia Aquática/UFCAR, São Carlos - SP, 2011.

THERMO ELECTRON CORPORATION.HiperToc, PreInstallation Guide. Netherlands: [s.n.], 2005.

TOMANOVA, S.; GOITIA, E.;HELESIC, J. Trophic levels and functional feeding groups of macroinvertebrates in neotropical streams. Hydrobiologia, v. 556, n. 1, p. 251-264, Feb. 2006.

YSEBART, T.; HERMANA, P. M. J.; MEIRE, P.; CRAEYMEERSCHD, J.; VERBEEKE, H.; HEIPA, C. H. R. Large-scale spatial patterns in estuaries: estuarine macrobenthic communities in the Schelde estuary, NW Europe. Estuarine, Coastal and Shelf Science, v. 57, n. 1-2, p. 335-355, May 2003. 